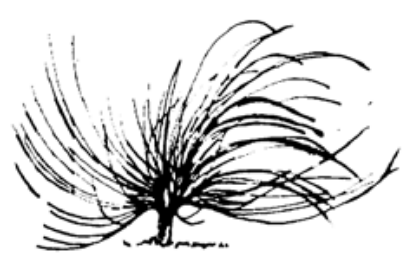

\title{
La biografía escolar como instrumento para la reflexión de los conocimientos previos y construidos durante la formación docente entorno al "cómo enseñar"
}

\author{
María Jesús Zárate-Montero ${ }^{l}$ \\ Universidad Nacional \\ Heredia, Costa Rica \\ mariajez@gmail.com
}

\begin{abstract}
Resumen
Este artículo es una revisión bibliográfica, cuyo propósito es analizar la narrativa como dispositivo de formación docente para el estudiantado universitario, por medio de la descripción y análisis de su biografía escolar, reflexione en torno a qué significa ser docente y al cómo y para qué enseñar. Los estudios consultados concluyen que esta tarea de aprendizaje puede desarrollarse con estudiantes que inician su carrera y con quienes realizan su práctica docente al finalizarla. La acción clave que se promueve es tomar conciencia de las concepciones construidas a lo largo de la vida sobre el papel y quehacer docente,
\end{abstract}

\section{(C) 1080}

Recibido: 2 de noviembre de 2015-Aprobado: 22 de setiembre de 2016

1 Educadora Musical y Máster en Educación con énfasis en Docencia Universitaria por la Universidad Nacional. Autora y coautora de artículos y ponencias relacionados con educación, educación musical, didáctica de la investigación, juegos cooperativos y acreditación de carreras universitarias. Académica e investigadora de la UNA desde 2007. 
y cómo estas pueden influir, en el actuar de las futuras y futuros educadores.

Palabras clave: Formación docente, dispositivos pedagógicos, narrativa, biografía escolar.

\begin{abstract}
This article is a literature review, which aims at analyzing narrative as a teacher training device for university students, which through the description and analysis of their school biography, reflects on what it means to be a teacher and how and what to teach. The consulted authors conclude that this task of learning can be developed with students starting their major and those doing their teaching practicum at the end of it. The key action to be promoted is being aware of conceptions built throughout life in the role and work of a teacher and how these can influence the actions of future educators.
\end{abstract}

Keywords: teacher training, pedagogical devices, narrative, school biography

\title{
Introducción:
}

Para iniciar este artículo, vale la pena tomar unos segundos y reflexionar sobre esta premisa:

Como afirmó Novak hace ya algún tiempo, muchos problemas educativos podrían resolverse mejor si pudiéramos comprender de manera fundamental cómo aprenden los niños, no es menos cierto que muchos problemas de la formación del profesorado podrían resolverse también mejor si comprendiéramos con mayor profundidad cómo aprenden profesores y profesoras a enseñar. (Montero, citado por Sanjurjo, 2012, p. 11)

Muchas veces, al iniciar un curso de formación docente, se suele preguntar al estudiantado sobre sus expectativas y conocimientos previos sobre el curso, pero pocas veces se les pregunta: ¿Por qué están estudiando para ser docentes?, y cuando se hace, la mayoría de las veces 
alguno o alguna levanta la mano y dice: "-Estoy en este curso mientras logro ingresar a la carrera que me gusta".

Pese a esta respuesta llena de sinceridad, conforme avanzan en los cursos, deciden quedarse y descubren la enseñanza de " $x$ " disciplina es lo suyo. En este caso, terminan felizmente su formación y, junto con colegas que siempre soñaron con ejercer, se inician en esta aventura.

En otros casos, el estudiantado manifiesta un gran interés por la docencia, pero al avanzar y tener contacto con la población de primaria o secundaria, concluye que este trabajo no es lo que espera, lo cual puede ser contradictorio; pero resulta más provechoso cambiar de dirección a tiempo que convertirse en un sujeto profesional más, que antes de empezar su labor se siente frustrado o frustrada.

En los casos descritos, su historia de vida antes y después de ingresar a la universidad fue determinante para decidir quedarse o cambiar de rumbo. Esto, porque a partir de las vivencias y aprendizajes en la familia y en el sistema educativo preuniversitario, tomamos decisiones sobre qué carrera deseamos estudiar y en el caso de la docencia iniciamos nuestros estudios con una idea sobre lo que significa enseñar y cómo enseñar, porque tenemos el recuerdo de aquellos y aquellas docentes que impactaron en nuestras vidas, ya fuera por sus buenas enseñanzas o por las que a nuestro parecer no fueron las más pedagógicas, didácticas o justas.

Es decir, la formación y quehacer docente están permeados de estos recuerdos y aprendizajes que se enriquecen y perfeccionan, siempre y cuando se reflexione sobre estos; de lo contrario, se corre el riesgo de reproducir aquello que no queremos.

Por esta razón, el narrar estas experiencias de nuestra biografía escolar resulta una experiencia de aprendizaje muy enriquecedora, porque permite contrastar nuestras concepciones con los conocimientos pedagógicos que vamos aprendiendo y a la vez reflexionar sobre cómo los estamos llevando a la práctica, para así tomar conciencia sobre si estamos ayudando a aprender o si seguimos replicando metodologías memorísticas y mecánicas.

De ahí que el propósito del presente trabajo es analizar la narrativa como dispositivo de formación docente para que el estudiantado universitario, a través de sus biografías escolares, reflexione sobre sus conocimientos previos y los que está construyendo en torno al ser docente y al cómo y para qué enseñar. 


\section{Estrategia para la búsqueda y selección de la información}

Para la presente investigación se establecieron las siguientes temáticas claves: dispositivos pedagógicos para la formación docente, narrativa y biografía escolar. Se consultaron libros y artículos de revistas académicas, los cuales fueron localizados mediante el catálogo del Sistema de Documentación de la Universidad Nacional (SIDUNA), Google académico y las bases Dialnet, Ebscohost, Scielo y Dipòsit Digital. La información recopilada fue revisada, seleccionada y organizada en los subtítulos que se desarrollan a continuación.

\section{Formación de docentes reflexivos}

En los planes de estudio de las carreras de formación docente, se contemplan una serie de conocimientos, destrezas y competencias que se espera desarrolle el estudiantado a lo largo de su formación.

Las actividades de aprendizaje que elijan sus profesores y profesoras deben tener como meta el comprender determinados contenidos para luego tomar decisiones y proponer posibles soluciones a los problemas educativos que estudian, en cada curso, con el fin de prepararles para los espacios de práctica docente en los que aplicarán sus conocimientos pedagógicos, didácticos y disciplinares al planear y desarrollar una clase.

Esta experiencia de empezar a enseñar le permite al profesorado en formación acercarse a los centros educativos, conocer sus realidades y comprender las dificultades propias del enseñar algo y el cómo se pueden mejorar sus prácticas para su desempeño futuro.

Para este proceso de preparar, ejecutar y revisar lo realizado, es decir, la reflexión de lo actuado, es necesario contar con dispositivos que orienten los aprendizajes que parten de la teoría, se lleven a la práctica y se analicen para generar nuevos conocimientos, de manera que se vayan perfeccionando.

$\mathrm{Al}$ respecto, Anijovich, Cappelletti, Mora y Sabelli, citadas por Schwartzman, Eder y Roni (2014) explican que:

Un dispositivo de formación docente es un modo particular de organizar la experiencia formativa con el propósito de generar situaciones experimentales para que los sujetos que participan 
en él se modifiquen a través de la interacción consigo mismos y/o con otros, adaptándose activamente a situaciones cambiantes, apropiándose de saberes nuevos, desarrollando disposiciones y construyendo capacidades para la acción. (p. 187)

En este sentido, quienes formamos docentes necesitamos indagar y aprender más sobre estas herramientas pedagógicas, con el fin de trabajarlas en nuestras clases, de manera que el estudiantado pueda tomar conciencia de sus concepciones previas sobre qué es ser educador o educadora, cómo enseñar, cómo evaluar o cómo mediar en el grupo.

Resulta necesario, entonces, revisar sus experiencias a lo largo de su vida, en especial aquellas vinculadas con su paso por el sistema educativo formal, es decir, describir y analizar eventos que forman parte de sus biografías escolares.

\section{La narrativa}

Sanjurjo (2012) plantea que la narrativa, al igual que otros dispositivos (investigación cualitativa, taller y ateneos didácticos), promueve el análisis y "el aprendizaje de una actitud reflexiva que posibilite un ejercicio permanente de compromiso, de revisión, de apertura" (p. 11).

Por esta razón, y tal como lo explica Ricoeur (2003, parafraseado por Caporossi, 2012), la narrativa reflexiva es un dispositivo que:

Al responder a un proceso metacognitivo, posibilita la interpretación de las acciones pedagógicas propias y públicas, psíquicas y culturales y a la par, explicitan el deseo de ser y el esfuerzo por existir que nos constituye como docentes.

En este sentido, es que sostenemos que la narrativa en la enseñanza y en el aprendizaje recupera la producción del sujeto, del que narra sobre su propia práctica de enseñanza y de aprendizaje frente a la imagen de reproducción o de pasividad. Es decir, que posibilita la recuperación de la autoría de las prácticas, el reconocimiento del proceso de construcción del conocimiento profesional docente y el mejoramiento de las prácticas de enseñanza y aprendizaje. (Caporossi 2012, pp. 112-113) 
Como puede observarse, a lo largo de su ejercicio profesional, el personal educativo construye y reconstruye saberes y, al narrarlos, puede tomar distancia para mirarlos desde diferentes perspectivas. Por esto, es primordial establecer tareas que ayuden al estudiantado, para que, de manera consciente, reflexione sobre los aprendizajes que está construyendo en sus prácticas docentes, por esta razón el profesorado que tenga a cargo dicho curso debe proponer:

Dispositivos de construcción del conocimiento profesional docente en los cuales los residentes puedan hablar de sus experiencias, compartirlas observarlas a posteriori, hacer preguntas, comprender por qué no comprenden, vivenciar el miedo a la exposición de la propia historia. (Cifali, citado por Coporossi, 2012, p. 121)

De igual manera, además de solicitar al estudiantado que narre sus experiencias y aprendizajes es importante que se abran espacios en la clase para releer, reflexionar, reconstruir y socializar lo aprendido en los procesos de práctica docente, de tal manera que se puedan obtener recomendaciones para futuras experiencias en las aulas.

\section{Biografía escolar, un dispositivo para aprender y reflexionar}

A continuación, se describen acciones realizadas por diferentes autores y autoras en las que trabajaron la biografía escolar con sus estudiantes de carreras de formación docente. Sus sugerencias son muy valiosas para quienes deseen implementar este dispositivo en sus cursos.

Pero antes de profundizar en estas propuestas es importante recordar que

la biografía escolar es un relato que refiere a los aprendizajes incorporados en el paso por la escolaridad, en todo ese trayecto en el que como sujetos estamos insertos en las instituciones educativas. En nuestras experiencias escolares, además de aprender los contenidos curriculares, interiorizamos otros saberes referidos a pautas de comportamientos, a cómo se aprende, cómo se estudia, cómo se enseña. $\mathrm{O}$ sea que, como producto de nuestra historia de alumnos, nos hemos apropiado de teorías, creencias, supuestos y 
valores sobre el conocimiento profesional docente. (Caporossi, 2012, p.113)

Estos saberes se constituyen en referentes tan fuertes como los estudiados durante la formación universitaria, de tal manera que se visibilizan en las decisiones y acciones del profesorado, tal y como lo explica Alliaud "uno puede aprender distintas corrientes pedagógicas, asumir incluso posturas dentro de las discusiones que existan en el campo del pensamiento didáctico, pero a la hora de enfrentar situaciones prácticas, concretas en el aula... sale lo que el cuerpo aprendió" (Citada por Caporossi, 2012, p. 115).

Por esta razón, la reconstrucción y análisis de estas experiencias es un punto de partida para aprender y desaprender aquellas concepciones sobre el quehacer docente.

Ahora bien, respecto a la implementación de este dispositivo, Fernández y Ramírez (2006) explican que en la cátedra Didáctica General del Centro Regional Universitario Bariloche:

[Se] solicita a los alumnos que reconstruyan una clase vivida en cualquiera de los niveles del sistema educativo, que resulte por alguna razón de interés, placentera o tensionante y que la describan detalladamente, desarrollando un relato escrito. Con los relatos individuales, se construye un texto colectivo que se utiliza como material de trabajo a lo largo del curso. (p. 1)

Estas investigadoras agregan que, por medio de esta tarea de aprendizaje, se genera un texto colectivo que al ser estudiado por el grupo promueve la metacognición en sus participantes, porque "objetiva el pensamiento de cada uno y lo hace público y discutible" (p. 3), es decir, el recuerdo se materializa en el escrito y este a su vez se convierte en tema de dialogo. Por ejemplo, entre las situaciones que suelen ser más frecuentes en las narraciones del estudiantado se encuentran:

- El clima afectivo de la clase y el vínculo docente-estudiante, como elementos constitutivos de la enseñanza que favorecen o entorpecen la tarea en el aula.

- El compromiso y la ética del profesorado con respecto a su tarea, vinculado con la motivación del alumnado que aprende. 
- La distribución y el uso del espacio como facilitadores u obstaculizadores de la enseñanza y el aprendizaje.

- La relación poder-conocimiento, asumida y otorgada por docentes y estudiantes.

- La relación teoría-práctica fragmentada, ocupando momentos bien diferenciados en las clases.

- Aspectos metodológicos vinculados con cuestiones ideológicas y con el concepto de curriculo oculto.

- Dimensión socio-política de la enseñanza poco considerada o al menos escasamente explicitada.

- Enfoques de enseñanza, reconocimiento de los elementos de cada uno de ellos. (Fernández y Ramírez, 2006, p. 4).

Como segundo ejemplo, Leite y Rivas (2013) describen que utilizan las biografías escolares del estudiantado de su facultad como punto de partida "para convertirlas en currículum de formación; esto es, el relato autobiográfico se convierte en el contenido de estudio colectivo para construir conocimiento y saberes educativos. Así, desde el compartir y relatarse mutuamente los relatos, la experiencia particular se convierte en conocimiento público, mediante el proceso de análisis e interpretación correspondiente" (p. 251).

Estos académicos parten de una pregunta central y el estudiantado, al estudiar sus respuestas, concreta dos caminos: uno interpretativo y otro conceptual. En el caso del primero "los alumnos y las alumnas van avanzando paulatinamente sobre las biografías, transformándolas a partir de los avances en el proceso de comprensión, y elaborando respuestas sobre la pregunta central que se apoyan en los sucesos biográficos" (pp. 253-254). En el segundo, "el proceso intenta buscar explicaciones acerca de los fenómenos que aparecen en las biografías, desde las tradiciones científicas y académicas correspondientes y la toma de postura ante ellas" (p. 254).

De esta manera, la tarea de aprendizaje "consiste en un continuo ir y venir entre ambos caminos con el ánimo de poder dar respuesta a la pregunta central y sobre la que tendrán que adoptar una posición teórica, práctica e ideológica" (p. 254).

En su artículo, Leite y Rivas (2013) describen ampliamente las fases para desarrollar su propuesta, la cual de manera resumida consiste en: 
1. Redactar las biografías anotando sus recuerdos más significativos.

2. Formar y trabajar en grupos para reflexionar y construir conocimientos.

3. Cada grupo contrasta sus biografías y se extraen temas que a su vez se comparan con los demás subgrupos de trabajo para elaborar de manera conjunta una nueva lista.

4. Sobre esta lista se plantean interpretaciones referentes a un concepto clave de la pregunta central.

5. Se categorizan y reducen las respuestas para estructurar un contenido que será un núcleo temático.

6. Con dicho núcleo se regresa a las biografías para extraer nueva información.

7. Cada estudiante reescribe su biografía desde una perspectiva crítica considerando las explicaciones que se generaron en la tarea.

8. Cada grupo realiza un trabajo para contestar la pregunta central.

Otra propuesta, similar a la anterior, es la descrita en la ponencia de Márquez et al. (2013), quienes explican la metodología que desarrollan como parte del proyecto "La biografía escolar como herramienta de aprendizaje, reflexión y crítica en la formación inicial del profesorado de educación primaria: BIOEDUCAMOS" (p. 1), en el que participan docentes y estudiantes que se forman como docentes de educación primaria, dicha "experiencia se desarrolla desde las asignaturas de organización de centros e instituciones educativas, investigación educativa en educación primaria, practicum I y III" (p.1).

En estos cursos, los grupos docentes participantes solicitan al estudiantado relatos sobre su biografía escolar antes de ingresar a la universidad, que luego serán analizados de manera grupal con el propósito de hacer una "confrontación de experiencias, sucesos, vivencias" (p. 3) que dan paso a:

... la búsqueda e identificación de ejes, núcleos o focos significativos que orientarán nuevas búsquedas de sentido hacia otros textos, teorías, perspectivas y experiencias que trascienden la experiencia escolar individual y grupal para avanzar hacia núcleos de sentido más profundos y a perspectivas de contexto más complejas. La experiencia busca la reconstrucción teórica y las 
teorías se convierten en herramientas posibles y diversas para la comprensión. (p. 3)

Los autores explican que en el proceso de análisis se utilizan diferentes estrategias para generar aprendizajes colaborativos que son presentados en formatos distintos "para dejar emerger otras narrativas sobre la escuela: dibujos, vídeos, representaciones, blogs, raps, comic, poemas, cuentos, pinturas" (p. 3), lo cual permite al estudiantado comunicar sus conclusiones en relación con ese ir y venir respecto al papel de la escuela y al qué significa ser docente.

$\mathrm{Al}$ respecto, Márquez et al. (2013) explican que este profesorado en formación realiza "análisis de diarios de clases, trabajo colectivo en wikis, para cada grupo y para la totalidad del grupo-clase, talleres de lectura de textos específicos, video fórum y seminarios sobre algunos focos o ejes de interés para el alumnado" (p. 3). En relación con los ejes mencionados, estos se originan "del análisis de los relatos [que] son analizados e interpretados una y otra vez desde diversos 'textos', diálogos, conceptos, teorías, perspectivas y poco a poco se van construyendo nuevos relatos sobre la escuela" (p. 3).

Como puede observarse, el analizar sus biografías escolares permite a estos grupos de docentes del futuro "repensar la escuela vivida y también la escuela deseada, la escuela en que quisieran trabajar, la escuela que les gustaría habitar" (p. 3).

Al concluir el curso, se consulta al estudiantado: “¿cómo valoran el proceso vivido?, ¿qué procesos de aprendizaje consideran que han transitado?, ¿con qué dificultades se han encontrado?, estos interrogantes constituyen algunas de las preguntas que se lanzan al final de la asignatura para continuar retroalimentando el proceso y para generar nuevas inquietudes" (pp.3-4).

Por otra parte y a diferencia de las experiencias descritas anteriormente, Delorenzi y Ruíz (2009) explican una metodología aplicada con estudiantes de un grupo de de la carrera Profesorado de Inglés (segundo año) y otro de Ciencias Biológicas (cuarto año) que realizaron su práctica docente. En ambos, compartieron la investigación educativa como un eje vertebrador, el cual se trabajó desde núcleos problemáticos: 
- El Profesorado de Inglés trabajó "su biografía escolar, y el impacto de ésta en la construcción de hipótesis para la futura práctica" (p. 4).

- $\quad$ El Profesorado de Ciencias Biológicas se concentró en "la biografía escolar, y el impacto de ésta en la construcción de la práctica durante el período de residencia" (p. 4).

Ahora bien, para el registro y análisis de los aprendizajes construidos en estos cursos se utilizó la biografía escolar como estrategia para "compartir la construcción de un relato y la argumentación teórica del mismo, el trabajo colaborativo, y el proceso de deconstrucción ideológica, teórica y práctica de los sujetos" (pp. 4-5) y el diario de cada docente como recurso que sirvió de "guía para la reflexión sobre la práctica, favoreciendo la toma de conciencia del profesor sobre su proceso de evolución y sobre sus modelos de referencia" (Porlán, citado por Delorenzi y Ruíz, 2009, p. 5).

Respecto al procedimiento desarrollado en los cursos, en el caso del Profesorado de Inglés, el estudiantado escribió sus biografías al inicio del curso, estas se analizaron y socializaron concentrando la atención en "aquellas enseñanzas implícitas que afloraron en las biografía" (Delorenzi y Ruíz, 2009, p. 5). Posteriormente, el grupo realizó una observación de aula y retomó las biografías para la elaboración de una hipótesis sobre "cómo implementar propuestas didácticas en la materia inglés en Educación Primaria Básica" (p. 5), para dicha tarea, además se elegir contenidos, "debían realizar una selección fundamentada de técnicas, estrategias, recursos didácticos y tecnológicos, y elaborar además propuestas de evaluación de los aprendizajes" (p. 5).

[Por su parte, el grupo de] profesorado en Ciencias Biológicas escribieron su biografía al comenzar el Espacio de la Práctica, y el diario del profesor al terminar cada una de las clases durante el período de residencia. El análisis de las biografías efectuado por el docente formador se socializaba por parejas de prácticas, y se contrastaba con lo escrito por cada alumno /a practicante, en la etapa pos activa de la residencia. Es decir, al culminar la residencia pedagógica, cuando cada pareja pedagógica realiza el proceso de deconstrucción de la práctica. (p. 5) 
Como consigna para ambos grupos, se estableció que para su biografía escolar narraran los "hechos del pasado escolar que los y las [sic] perfilen como sujetos escolares en los diferentes niveles educativos, incluso de la formación docente" (p. 6).

Además, a estudiantes de Ciencias Biológicas se les indicó que para la redacción del diario de clase describieran y analizaran lo acontecido en cada sesión; en dicho trabajo debían considerar "aspectos referidos a cómo enseñaron, cómo aprendieron los alumnos, cómo evaluaron, estrategias, material didáctico, lugar del practicante en el aula, cuestiones normativas, disciplina, entre otros" (p. 6).

De esta experiencia, Delorenzi y Ruíz (2009) concluyen que en el caso del grupo de Inglés, las biografías escolares evidencian "enseñanzas implícitas sobre qué entienden por enseñar, aprender y evaluar" (p. 6), lo que a su vez influyó en la preparación de la hipótesis que realizaron. Esta situación también se observó con el estudiantado de Ciencias Biológicas, tanto al planear cómo desarrollar sus clases (las cuales fueron expositivas, sin participación de los aprendientes y con una evaluación memorística), y por medio del diario de clase se constató su dificultad para problematizar su propia práctica pedagógica.

Como propuesta similar, Caporossi (2012) plantea que al estudiantado residente de su facultad, es decir, los grupos que realizan su práctica docente, se les solicitan dos trabajos reflexivos, uno al iniciar y el otro al concluir dicha práctica; además, con el propósito de facilitar el recuento cronológico y dar coherencia a la narración, desarrollan dos guías, las cuales se presentan a continuación.

\section{Trabajo práctico N. ${ }^{\circ} 1$}

Elaborar un relato biográfico "integrado" sin diferenciar cada aspecto solicitado. Las dimensiones intentan captar las referencias constitutivas del recorrido biográfico personal. Fundamentar los comentarios:

- Dimensión personal. Datos biográficos generales.

- Dimensión institucional: a) Escolaridad. b) Profesión (para quienes son docentes).

- Dimensión socio-política.

- Dimensión proyectiva. 


\section{Trabajo práctico N. ${ }^{\circ} 2$}

Realizar una narrativa personal teniendo en cuenta los siguientes ítems, ayudándose con la lectura del trabajo práctico N. ${ }^{\circ} 1$.

¿Cómo cree que influyó su biografía escolar en su práctica docente realizada en la residencia? ¿Cómo cree que influye en su formación general? ¿Cree que su biografía lo ayuda a construirse como docente de Ciencias de la Educación? En caso de respuesta afirmativa: ¿De qué forma?¿Puede identificar algunas cuestiones de su biografía escolar que aparecieron en el desarrollo de sus clases como residente, tanto potenciadoras como obturantes? ¿Cuáles? ¿Ha detectado actitudes puestas en acto en su práctica preprofesional que no pudo modificar y que tienen una relación directa con los modelos internalizados de su biografía escolar? Proponga estrategias para modificar las dificultades que surgieron en sus clases, producto de los modelos internalizados acríticamente. (p. 117)

De este proceso reflexivo, el estudiantado construye nuevos aprendizajes para su futura labor docente, por ejemplo, las narraciones que elaboran se analizan en subgrupos dirigidos por sus docentes "haciendo asociaciones, recordando detalles olvidados, reconociendo y antes y un después" (p. 121). Es así como el estudiantado reconoce "el impacto que tienen las situaciones escolares y los modelos docentes internalizados durante su trayectoria escolar en la manera de pensar, mirar y resolver situaciones de su experiencia de residencia” (p. 121).

Para cerrar este recuento de propuestas metodológicas relacionadas con la biografía escolar como dispositivo pedagógico para la formación de docentes, es importante destacar que esta como estrategia metacognitiva dirige al profesorado en formación a reflexionar sobre: ¿cómo planea su clase y la desarrolla?, ¿cómo interactúa con sus estudiantes?, ¿cómo evalúa sus aprendizajes?, ¿qué aprende de cada clase?, ¿cómo se mira a sí mismo o a sí misma?, ¿qué temores tiene?,¿cómo se siente?, ¿qué desaprende?, ¿qué sigue reproduciendo?

Justamente por esta última inquietud, resulta indispensable que este ejercicio reflexivo se pueda considerar como un eje en la formación de docentes, porque el tomar consciencia de los saberes y concepciones 
sobre el "cómo enseñar" puede ser un medio para que este estudiantado comprenda que tiene en sus manos el conocimiento y el poder para generar oportunidades de aprendizajes críticos, creativos y significativos para las personas que ayudará a aprender o que, lastimosamente, puede convertirse en un ente reproductor acrítico de los modelos que vivió antes de formarse como docente.

\section{Conclusiones}

La preparación profesional de un personal docente reflexivo requiere de la implementación y seguimiento de tareas de aprendizaje que propicien espacios de reflexión y diálogo, que partan de un análisis guiado de las experiencias personales que influyeron en su decisión de estudiar una carrera vinculada a la docencia.

Las propuestas analizadas en este artículo concluyen que la narrativa es un medio para reconstruir la biografía escolar, la cual puede trabajarse en todos los niveles de las carreras de formación docente. Sugieren, además, procedimientos para trabajar este dispositivo de la mejor manera posible, por ejemplo, iniciar con un relato individual sobre los "saberes pedagógicos" que construye cada estudiante antes de ingresar a una carrera de enseñanza, del cual surgen sus concepciones sobre lo que significa enseñar, aprender y ser docente, entre otras.

De esta primera etapa se debe pasar a la reflexión colectiva para contrastar estos conocimientos con los del resto del grupo, del profesorado y la teoría que se estudia en los cursos. Esto, aunado a experiencias de práctica pedagógica en instituciones educativas, obliga al estudiantado a reflexionar nuevamente sobre su aprendizaje, primero en solitario y luego con sus pares y docentes.

Este ir y venir se trata de un desaprender para aprender, con el propósito de lograr una congruencia entre nuestras acciones, creencias, conocimientos y valores.

En este camino, estamos llamados a sopesar nuestro actuar para identificar modelos internalizados acríticamente que, pese a los años de formación, se resisten a ser modificados y se siguen reproduciendo mecánicamente, aunque no son los más adecuados.

Tanto en nuestra práctica de formar educadoras y eduadores, como el estudiantado que participa de este proceso tenemos que tomar conciencia de esto, para proponer cambios en nuestro quehacer pedagógico de tal manera que las nuevas generaciones puedan recordar docentes con 
actitudes críticas y creativas que, con su discurso y sus acciones, evidenciaron que enseñar es ayudar a aprender de manera significativa.

\section{Referencias}

Delorenzi, O. y Ruiz, M. (2009). Narrativa y construcción de la práctica: Un modo de analizarla y redefinirla. En L. Porta, Z. Álvarez, M. Sarasa y S. Bazán (Comps.), Actas de las "V Jornadas sobre la Formación del Profesorado: Docentes, narrativas e investigación educativa". Recuperado de http://www.mdp.edu.ar/humanidades/cienciasdelaeducacion/jornadas/profesorado2009/final/ comunicaciones/2_narrativas/2a/2a_09.pdf

Caporossi, A. (2012). La narrativa como dispositivo para la construcción del conocimiento profesional de las prácticas docentes. En L. Sanjurjo, Los dispositivos para la formación en las prácticas profesionales. Rosario: Homos Sapiens Ediciones.

Fernández, M. y Ramírez, P. (enero 2006). Los relatos de experiencias escolares en la formación docente. Revista Iberoamericana de Educación, 37(4). Recuperado de http://www.rieoei.org/deloslectores/1131Fernandez.pdf

Leite, A. y Rivas, J. (2013). La biografía escolar en la formación del profesorado. En A. Lopes, F. Hernández, J. M. Sancho y J. I. Rivas (Coords.), Histórias de vida em Educação: a Construção do Conhecimento a partir de Histórias de Vida. Barcelona: Universitat de Barcelona. Dipòsit Digital. Recuperado de http://diposit. ub.edu/dspace/handle/2445/47252

Márquez, M., Rivas J., Leite, A., Cortés, P., Prados, E. y Padua, D. (2013). Los relatos escolares en la formación inicial: Procesos, paradojas y desafíos. En Simposio internacional: Aprender a ser docente en un mundo en cambio, 21 - 22 de noviembre. Recuperado de http://som.esbrina.eu/aprender/docs/6/MarquezGarciaMariaJesusPradosMegiasEstheryPaduaArcosDaniela.pdf

Sanjurjo, L. (Coord.). (2012). Los dispositivos para la formación en las prácticas profesionales. Rosario: Homos Sapiens Ediciones.

Schwartzman, G., Eder, M. y Roni, C. (septiembre - diciembre 2014). Formación docente en y para la universidad: Dispositivos y prácticas en ciencias de la salud. REDU. Revista de Docencia Universitaria, 12(4),179-201. Recuperado de http://red-u.net/redu/ index.php/REDU/article/view/700 